ARTICLE TEMPLATE

\title{
Intelligent tracking of moving ships in constrained maritime environments using AIS
}

\author{
Yuanchang Liu, Rui Song and Richard Bucknall \\ Department of Mechanical Engineering, University College London, Torrington Place, London WC1E \\ 7JE, UK
}

\section{ARTICLE HISTORY}

Compiled July 4, 2019

\begin{abstract}
With the increasing interest in autonomous shipping technology, to ensure ship's safety it is vital to equip the ship with a navigational algorithm to continuously track the moving vessels in the area of interest and subsequently avoid any collisions. To address this problem, in this paper an intelligent and robust tracking algorithm using Kalman filter and Interacting Multiple Model (IMM) scheme has therefore been designed. The algorithm is able to provide accurate motion information of other ships in constrained environments using least navigational information. The capability of the proposed algorithm has been tested and verified in a number of computer based simulations.
\end{abstract}

\section{KEYWORDS}

Interacting multiple model (IMM), Kalman filter, Maritime navigation, Automatic identification system (AIS)

\section{Introduction}

Autonomous shipping is the future for the maritime industry involving a number of different evolutionary technologies. To ensure the success of autonomous shipping, navigational safety has become the priority and it is important for the ship to autonomously detect other vessels during 
the operation and consequently take proper evasive actions. To detect other ships' movements, various navigational sensors such as the Autonomous Identification System (AIS), marine radar, and the Light Detection and Ranging (LIDAR) can be used. Among them, AIS is commonly used as it is fitted to all vessels over 300 gross tonnage (GT) and to many small sized vessels to enhance the safety and efficiency of maritime navigation according to the International Maritime Organisation (IMO) recommendations. The benefits of using the AIS are: 1) the coverage of the AIS information is at least 15 - 20 nautical miles (Arroyo 2011); 2) the information provided by the AIS is more comprehensive, including maritime mobile service identity (MMSI) number, vessel name, vessel position, speed and etc; 3) since AIS transmits on VHF radio frequency, it has the capability to continue to be used to detect other ships even in severe environments where the radar detection is compromised (Harati-Mokhtari et al. 2007).

It remains problematic however to access accurate and reliable information via the AIS, especially the dynamic information such as the instantaneous position and velocity. In essence, the AIS is a transmission broadcasting ship information to surrounding vessels. The information transmitted by the AIS comprises both the static information such as the MMSI number and vessel type, and the dynamic information such as the position provided by the GPS and the heading from the electronic compass. The dynamic information can be compromised by the devices' functionality such as the data unreliability through inaccuracy. For example, in terms of the ship's position, under normal operations, the data accuracy level is normally set as $10 \mathrm{~m}$; whereas, such an accuracy can be impaired by severe weather conditions (Harati-Mokhtari et al. 2007).

Another important issue associated with the AIS is data inconsistency. AIS transmits the dynamic information at different update rates according to a vessel's speed (shown in Table 1). The AIS data transmission mechanism is represented in Figure 1. It consists of two sections, i.e. the data receiving point (DRP) and the data waiting period (DWP). Navigation information is transmitted at each $D R P$; whereas, no information is available during $D W P$. In general, for reasons of safety, a high speed ship has higher updating rate and shorter $D W P$. However, it has been reported that AIS information is sometimes partially or fully interrupted; meaning, it is not received properly (Last et al. 2014). The data loss rate may be worse in regions with dense traffic due to 'data jamming' problems because of the limited bandwidth of transmission channel (Liu and Chen 2013).

The aforementioned problems associated with the AIS have direct influence on the safety of maritime navigation, it is therefore important and necessary to ensure the received data is processed 


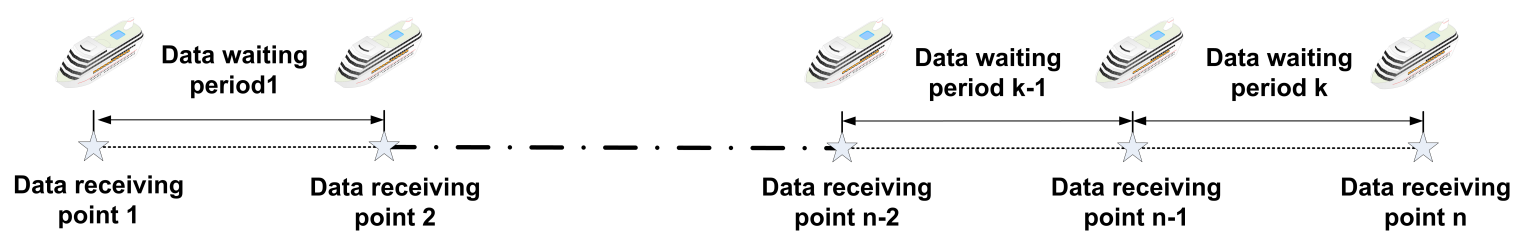

Figure 1. AIS broadcasting mechanism. The transmission consists of two different periods. At the data receiving point, AIS information is received and updated. During data waiting period, AIS information is absent.

Table 1. Report rate of dynamic information of AIS.

\begin{tabular}{ll}
\hline Type of ship & General reporting interval \\
\hline Ship at anchor & $3 \mathrm{~min}$ \\
Ship 0-14 knots & $12 \mathrm{~s}$ \\
Ship 0-14 knots and changing course & $4 \mathrm{~s}$ \\
Ship 14-23 knots & $6 \mathrm{~s}$ \\
Ship 14-23 knots and changing course & $2 \mathrm{~s}$ \\
Ship >23 knots & $3 \mathrm{~s}$ \\
Ship >23 knots and changing course & $2 \mathrm{~s}$ \\
\hline
\end{tabular}

to provide high accuracy information. The processing system should be capable of predicting the trajectory of the moving ship when data is lost. In this paper, a new algorithm has been developed for intelligent tracking of moving ships in constrained maritime environment. According to the dynamic characteristics of marine vessels, Kalman filter has been applied as the core for the algorithm and the Interacting Multiple Model (IMM) is employed to deal with more complicated moving motions. The algorithm have been verified in a series of simulations to test its capabilities.

\section{Trajectory tracking of a moving ship based on AIS}

Based on the work in Liu et al. (2017), an improved Kalman Filter based trajectory tracking algorithm (KFTTA) has been proposed to increase the accuracy of AIS information regardless of whether transmissions are regular or irregular. There are two processes composing the KFTTA namely the Updating Process and the Predicting Process (Figure 2). At each DRP, information including velocity, position and navigational status can be received but with a degree of uncertainty through sensors' inaccuracy. The Updating Process works by using the KF algorithm to increase the accuracy of the AIS information. During the $D W P$, no information is exchanged, and the Predicting Process is employed to provide an estimation of moving ships by using the prediction function of the KF (or the dead reckoning algorithm). The Updating Process and the Predicting Process will now be explained in detail. 


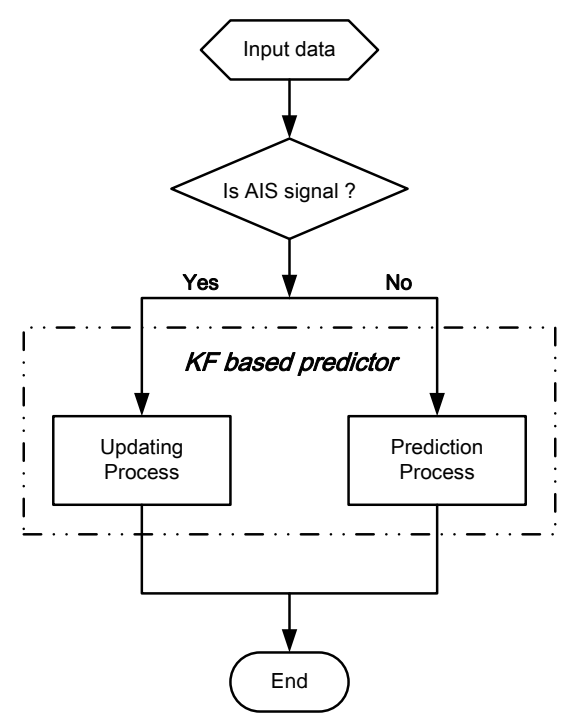

Figure 2. KF based trajectory tracking algorithm.

\subsection{The Kalman filter (KF) algorithm}

As a linear recursion algorithm, the $\mathrm{KF}$ is able to estimate the state of a dynamic system by following the least mean square error principle. The KF algorithm is summarised in Figure 3, which consists of two main processes namely the Time Update Process and the Measurement Update Process. The recursive estimation process of the KF mainly involves two different equations, i.e. the state transition equation and the measurement equation:

$$
X_{k+1}=A_{k} X_{k}+B_{k} u_{k}+w_{k}
$$

$$
Z_{k}=H_{k} X_{k}+v_{k}
$$

where in Eq. $1 X_{k}$ is the system state vector at time $k, A_{k}$ is the state transition matrix and $B_{k}$ is the control input matrix applied on the control input $u_{k}$. In Eq. $2, Z_{k}$ is the system observation vector at time $k$ and $H_{k}$ is the observation matrix. $w_{k}$ and $v_{k}$ are the transition noise and observation noise respectively. 


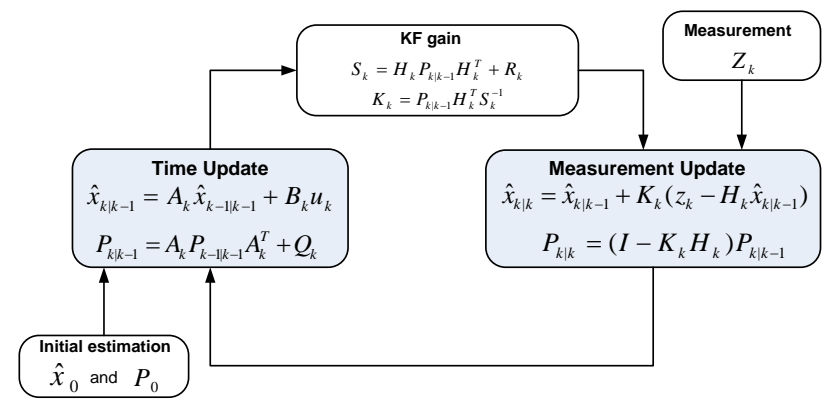

Figure 3. The recursive process of the Kalman Filter.

\subsection{Motion update of moving ship based on the KF}

When implementing the KF for a position update in the maritime environment, the system state vector can be expressed as:

$$
X=\left[x, y, v_{x}, v_{y}\right]^{T}
$$

where $x$ and $y$ represent the position in the $\mathrm{x}$ direction and y directions, $v_{x}$ and $v_{y}$ are velocities in the $\mathrm{x}$ and $\mathrm{y}$ directions. A motion model to represent the movements of a ship is required to facilitate the implementation of the KF. Since the majority of marine vessels are neither highly manoeuvrable nor change their speed rapidly within certain sampling times (Campbell et al. 2014), it is therefore reasonable and feasible to represent the motion of ships by using the motion representation, which consists of:

- Straight line motion model: A model of the straight line movement;

- Circle motion model: A model of the course change movement.

To describe mathematically these motions, two motion models, i.e. the constant velocity model (CVM) and the coordinated turn model (CTM), have been employed respectively.

\subsubsection{Ship motion based on the constant velocity model (CVM)}

In the constant velocity model (CVM), it is assumed that the velocity $v(k)$ has a constant value during the $k^{\text {th }}$ sampling period $(\Delta T)$. However, in practice, the velocity is likely to suffer small perturbations caused by the ocean environment such as the wind and current, which can be modelled using a zero-mean white noise sequence. Hence, the discretised ship's movement equation 
based on the CVM can be written as:

$$
X_{k+1}=A_{C V M} X_{k}+G_{k} a_{k}
$$

with

$$
A_{C V M}=\left(\begin{array}{cccc}
1 & 0 & \Delta T & 0 \\
0 & 1 & \Delta T & 0 \\
0 & 0 & 1 & 0 \\
0 & 0 & 0 & 1
\end{array}\right)
$$

and

$$
G_{k}=\left(\begin{array}{cc}
\frac{\Delta T^{2}}{2} & 0 \\
0 & \frac{\Delta T^{2}}{2} \\
T & 0 \\
0 & T
\end{array}\right)
$$

where $\Delta T$ is the sampling period and $a_{k}$ is defined as:

$$
a_{k} \sim N\left(0, \sigma^{2}\right)
$$

which is a zero-mean white noise used to model the uncertain accelerations in $\mathrm{x}$ and $\mathrm{y}$ directions. Now, Eq. 4 can be rewritten as:

$$
X_{k+1}=A_{C V M} X_{k}+w_{k}, \quad w_{k} \sim N\left(0, Q_{C V M}\right)
$$

with

$$
Q_{C V M}=E\left[G_{k} a_{k} a_{k} G_{k}^{T}\right]=\sigma^{2}\left(\begin{array}{cccc}
\frac{\Delta T^{4}}{4} & 0 & \frac{\Delta T^{3}}{2} & 0 \\
0 & \frac{\Delta T^{4}}{4} & 0 & \frac{\Delta T^{3}}{2} \\
\frac{\Delta T^{3}}{2} & 0 & \Delta T^{2} & 0 \\
0 & \frac{\Delta T^{3}}{2} & 0 & \Delta T^{2}
\end{array}\right)
$$


Since only the position coordinates of the moving ship need to be estimated, the measurement (observation) vector $Z_{k}$ is now in the form of $[x, y]^{T}$, and Eq. 2 can be written as:

$$
\left(\begin{array}{l}
x(k) \\
y(k)
\end{array}\right)=\left(\begin{array}{cccc}
1 & 0 & 0 & 0 \\
0 & 1 & 0 & 0
\end{array}\right)\left(\begin{array}{c}
x(k) \\
y(k) \\
v_{x}(k) \\
v_{y}(k)
\end{array}\right)+v_{k}
$$

where $v_{k}$ is used to simulate the measurement noise, which is expressed as a Gaussian white noise with zero mean and standard deviation $\sigma_{z}$.

\subsubsection{Ship motion based on the coordinated turn model (CTM)}

The CVM model is established on the random process (or Gaussian process), which is not capable of modelling more complex ship manoeuvres such as turning. Therefore, the coordinated turn model (CTM) has been proposed to model such a manoeuvre based upon the ship's kinematics.

When a ship is making a turn with a constant transverse velocity of $V$ at time step $t$, the standard curvilinear-motion can be used to describe ship motion as:

$$
\begin{array}{r}
\dot{x}(t)=V(t) \cos \phi(t) \\
\dot{y}(t)=V(t) \sin \phi(t) \\
\dot{V}(t)=a_{t}(t) \\
\dot{\phi}(t)=\frac{a_{n}(t)}{V(t)}
\end{array}
$$

where $x(t), y(t)$ and $\phi(t)$ are the position and heading of ship at time step $t, a_{n}(t)$ and $a_{t}(t)$ denote the ship's normal and tangential accelerations (Li and Jilkov 2000).

Based on Eq. 11, there are three different cases that can be deduced as:

- $a_{n}(t)=0, a_{t}(t)=0$ : rectilinear and constant speed motion;

- $a_{n}(t)=0, a_{t}(t) \neq 0$ : rectilinear and accelerating motion;

- $a_{n}(t) \neq 0, a_{t}(t)=0$ : circular and constant speed motion.

The last case is named as the coordinated turn model (CTM) if constant speed and turn rate have 
been implemented.

Assume the motion of the ship is still represented by the state vector as Eq. 3 and the ship has the constant speed $V$ and the constant angular rate $\omega$, the continuous circular motion equation of the ship is:

$$
\dot{x}(t)=F(\omega) x+w(t), \quad F(\omega)=\left(\begin{array}{cccc}
0 & 0 & 1 & 0 \\
0 & 0 & 0 & 1 \\
0 & 0 & 0 & -\omega \\
0 & 0 & \omega & 0
\end{array}\right)
$$

where $F(\omega)$ is the system transition matrix. By using the system discretisation approach in (Bar-Shalom et al. 2004), matrix $F(\omega)$ can be discretised to $A_{C T M}(\omega)$ as:

$$
A_{C T M}(\omega)=e^{F(\omega) \Delta T}=\left(\begin{array}{cccc}
1 & 0 & \frac{\sin (\omega \Delta T)}{\omega} & -\frac{1-\cos (\omega \Delta T)}{\omega} \\
0 & 1 & \frac{1-\cos (\omega \Delta T)}{\omega} & \frac{\sin (\omega \Delta T)}{\omega} \\
0 & 0 & \cos (\omega \Delta T) & -\sin (\omega \Delta T) \\
0 & 0 & \sin (\omega \Delta T) & \cos (\omega \Delta T)
\end{array}\right)
$$

And Eq. 12 can be discretised as:

$$
X_{k+1}=A_{C T M}(\omega) X_{k}+w_{k}
$$

where $w_{k}$ is a $4 \times 1$ zero-mean Gaussian vector modelling the perturbation of the trajectory with the covariance of:

$$
Q_{C T M}=\left(\begin{array}{cccc}
q_{x}^{2} & 0 & 0 & 0 \\
0 & q_{y}^{2} & 0 & 0 \\
0 & 0 & q_{v x}^{2} & 0 \\
0 & 0 & 0 & q_{v y}^{2}
\end{array}\right)
$$

where $q_{x}$ and $q_{y}$ denote the perturbations in $\mathrm{x}$ and $\mathrm{y}$ direction respectively. The measurement equation for the CTM is the same as the CVM given by Eq. 10. 


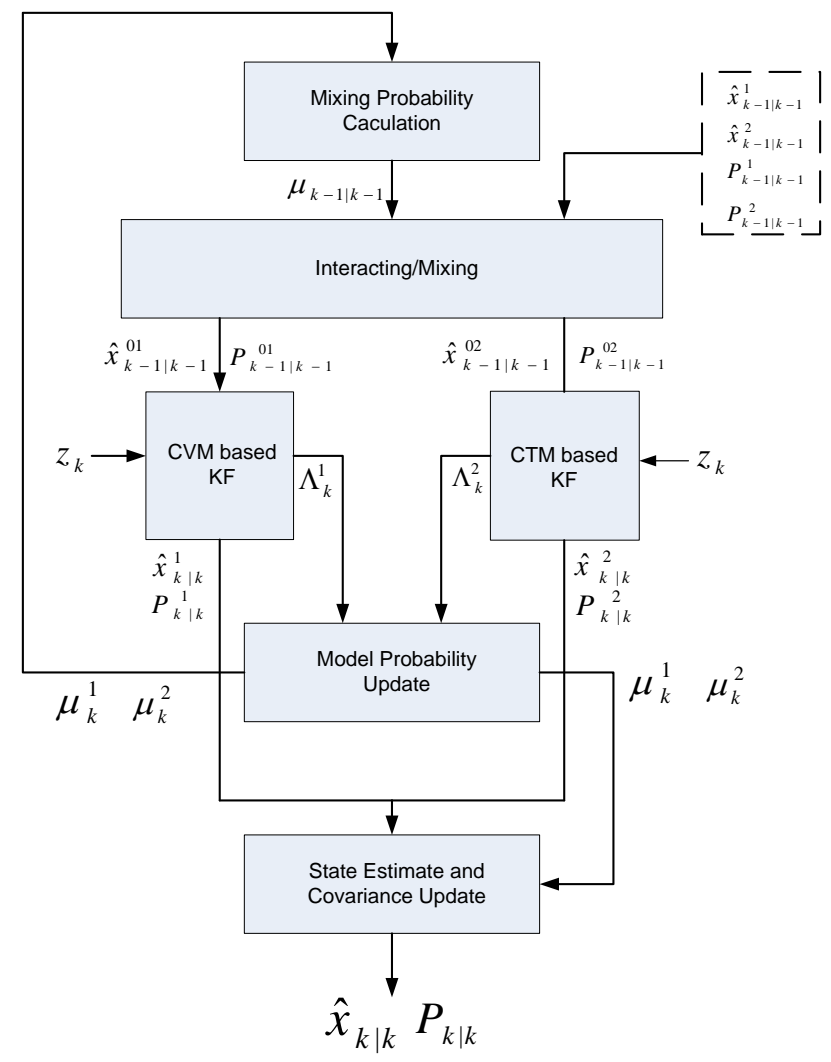

Figure 4. The IMM-filtering for CVM and CTM combined model (one cycle).

\subsubsection{Interacting Multiple Model (IMM) filter for model integration}

In fact, a more realistic ship navigation pattern is the combination of both the CVM and CTM. This is because the positions of moving ships cannot be precisely determined by either model. Hence, the Interacting Multiple Model (IMM) filtering (IMM-filter) has been implemented. The IMM-filter was first proposed by Bar-Shalom et al. (2004) for such filtering problems where multiple models are used. This can be viewed as a Markovian switching system characterised by the model transition probabilities. The IMM-filter is able to provide the suboptimal estimation of the system by calculating the weighted combination of the estimation of each model.

Figure 4 illustrates the designed IMM-filter for the CVM and the CTM combined ship motion model. Suppose the CVM and the CTM are denoted as Model 1 and Model 2. For one operation cycle (at time step $k$ ), the IMM-filter first calculates the mixing probability of the two models 
based upon the probability of each model as calculated in the previous time step as:

$$
\begin{aligned}
\mu_{k-1 \mid k-1}^{i \mid j} & =\frac{1}{\overline{c_{j}}} p_{i j} \mu_{k-1}^{i}, \quad i, j=1,2 \\
\overline{c_{j}} & =\sum_{i=1}^{2} p_{i j} \mu_{k-1}^{i}, \quad j=1,2
\end{aligned}
$$

where $p_{i j}$ is the probability of switching from Model $i$ to Model $j$ in the next time step and $\mu_{k-1}^{i}$ is the probability of Model $i$ at time step $k-1$.

The mixed inputs for CVM based KF and CTM based KF can then be calculated by referring to the mixing probability as:

$$
\begin{array}{r}
\hat{x}_{k-1 \mid k-1}^{0 j}=\sum_{i=1}^{2} \hat{x}_{k-1 \mid k-1}^{i} \mu_{k-1 \mid k-1}^{i \mid j}, \quad j=1,2 \\
P_{k-1 \mid k-1}^{0 j}=\sum_{i=1}^{2} \mu_{k-1 \mid k-1}^{i \mid j} \times\left\{P_{k-1}^{i}+\left[\hat{x}_{k-1 \mid k-1}^{i}-\hat{x}_{k-1 \mid k-1}^{0 j}\right]\left[\hat{x}_{k-1 \mid k-1}^{i}-\hat{x}_{k-1 \mid k-1}^{0 j}\right]^{T}\right\}, \quad j=1,2
\end{array}
$$

By using the measurement $Z(k)$ and the calculated mixed inputs $\left(\hat{x}_{k-1 \mid k-1}^{0 j}, P_{k-1 \mid k-1}^{0 j}\right)$, ship's state and covariance are updated using two Kalman Filters respectively as:

$$
\left[\hat{x}_{k \mid k}^{j}, P_{k \mid k}^{j}\right]=K F\left(\hat{x}_{k-1 \mid k-1}^{0 j}, P_{k-1 \mid k-1}^{0 j}, Z(k), A^{j}, H^{j}, Q^{j}, R^{j}\right), \quad j=1,2
$$

And the likelihood of the measurement of each filter is:

$$
\Lambda_{k}^{j}=N\left(v_{k}^{j} ; 0, S_{k}^{j}\right), \quad j=1,2
$$

where $v_{k}^{j}$ is the measurement residual and $S_{k}^{j}$ is the covariance for Model $j$ at time step $k$. The likelihood of each filter $\left(\Lambda_{k}^{j}\right)$ can be interpreted as the confidence level of current filter, i.e. small measurement residual will produce a high likelihood of the current filter meaning the filter is more accurate and should be more relied upon at that moment. Therefore, the probabilities of 
each model at time step $k$ can be calculated as:

$$
\begin{array}{r}
\mu_{k}^{j}=\frac{1}{c} \Lambda_{k}^{i} \bar{c}_{j}, \quad j=1,2 \\
c=\sum_{j=1}^{2} \Lambda_{k}^{j} \bar{c}_{j}
\end{array}
$$

Finally, the combined estimation of the ship's state and covariance at time step $k$ can be obtained by:

$$
\begin{array}{r}
\hat{x}_{k \mid k}=\sum_{i=1}^{2} \mu_{k}^{i} \hat{x}_{k \mid k}^{i} \\
P_{k \mid k}=\sum_{i=1}^{2} \mu_{k \mid k}^{i} \times\left\{P_{k \mid k}^{i}+\left[\hat{x}_{k \mid k}^{i}-\hat{x}_{k \mid k}\right]\left[\hat{x}_{k \mid k}^{i}-\hat{x}_{k \mid k}\right]^{T}\right\}
\end{array}
$$

\subsection{Motion prediction of moving ship}

During the transmission interval (the $D W P$ ), the perception of the moving ship's positions is still important to ensure the safe navigation of a USV. However, because there is no accurate information established during this period, the USV can only make a prediction of a ship's movement information. The prediction process of the KF algorithm loop (Figure 3) is repeatedly executed to calculate the possible positions of moving ship from time step $k$ to $k+1$ based on the latest known information as:

$$
X_{k+1}=A_{k} X_{k}
$$

where $A_{k}$ is the motion model currently being used by the ship. Such a prediction process has no corrections and will possibly accumulate large errors if the prediction time is too long. Therefore, as new moving ship's information is received the full KF loop should continue to be employed to compensate for the generated errors.

Eq. 22 can also be iterated for $n$ time steps in one calculation cycle, and the generated results will then consist of a series of positions indicating the possible trajectory for next $n$ time steps of the moving ship. 


\section{Simulations and discussions}

In this section, simulations have been carried out to validate the capability of the proposed KFTTA of tracking moving ships using only AIS information. Simulations are structured based on different ship motion models as: 1) ship based on the CVM to model the straight line motion, 2) ship based on the CTM to model the course changing motion and 3) ship based on the CVMCTM combined motion to model a complex ship manoeuvre.

\subsection{Trajectory tracking based on the CVM}

Ship trajectory tracking based on the CVM has been tested in this section. It is assumed that the moving ship starts its motion from position $(0,0)$ with a speed of 4 knots and heading angle of $26^{\circ}$. The ship is suffering from the system noise, which can be modelled by a Gaussian white noise sequence (Eq. 7) with zero mean and variance of $\sigma=1 \mathrm{~m} / \mathrm{s}^{2}$. The navigation information such as the ship's location and velocity is transmitted and reported through AIS. The instantaneous position coordinates are used as the measurement information for the KF. Also the measurement is associated with noise, which is a Gaussian white noise with zero mean and covariance diag $\{1,1\} \times 10^{2}$ representing an accuracy of the AIS position information as $10 \mathrm{~m}$.

According to the Table 1, a ship travelling at 4 knots has the AIS update rate of $4 \mathrm{~s}$. However, to test the prediction capability of the proposed algorithm, in this simulation it is assumed the ship is travelling in a harsh environment which interrupts its normal AIS transmission and the new AIS update rate is configured as $60 \mathrm{~s}$. Therefore, the algorithm needs to make a prediction of the ship motion during this time period. For this simulation the prediction time is set as every $12 \mathrm{~s}$.

Simulation results showing how the vessel's position information is updated and predicted in 15 min are presented in Figure 5. As the simulation covers a time period of 15 min, 15 consecutive data-receiving points are plotted with the vessel's true position drawn as black square markers and the inaccurate measured position as red circle markers. Positions obtained using the KF are plotted with blue star markers. From Figure 5a, it can be observed that the KF can increase the accuracy by providing truer position points. Such an observation can be further verified in Figure $5 \mathrm{~b}$, which shows the position errors of measurement data (plotted as blue line) and errors of the data filtered by the KF (plotted as red line). It is evident the error of the KF is much smaller than the error of the measurement as the red line is well below the blue line with the exception of time steps $11 \mathrm{~min}$ and $14 \mathrm{~min}$. During these two time steps, the measurement provides more 


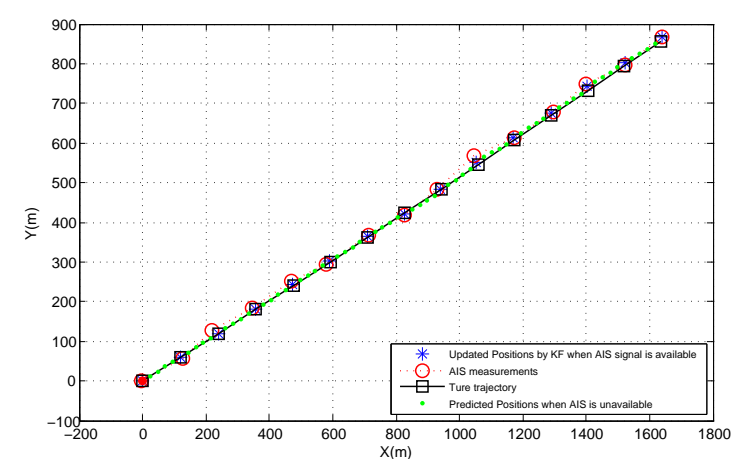

(a)

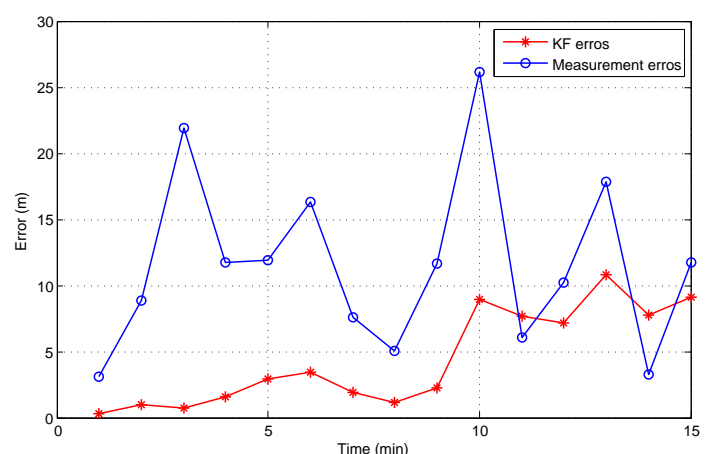

(b)

Figure 5. Simulation results of the trajectory tracking using the proposed KFTTA based on CVM. (a) Simulation results with true positions plotted with black square markers, observed positions plotted with red circle markers, positions provided by the KF plotted with start markers and predicted positions with green dot markers. (b) Comparison of position errors of measurement data and the data produced by the KFTTA.

accurate information as the measurement errors are about $6 \mathrm{~m}$ and $3 \mathrm{~m}$ and $\mathrm{KF}$ errors are $7 \mathrm{~m}$ and $8 \mathrm{~m}$, respectively.

In terms of the position prediction when the AIS information is unavailable, the predicted results are plotted with green dot markers in Figure 5a. It can be observed that the predicted positions stay close to the true trajectory of the vessel, which means that the predictor is able to continue to provide the vessel's position even though the AIS data is absent.

\subsection{Trajectory tracking based on the CTM}

The CTM model has been used in this section to validate the capability of the algorithm for tracking and predicting the motion of a ship when making a course change. The ship starts from the point of $(250,250)$ with the velocity of 6 knots and $90^{\circ}$ course heading. The turning rate of the ship is 15 degrees/min. The motion is subjected to a noise (Eq. 15) modelled by a Gaussian white noise sequence with zero mean and the variance $q_{x}=q_{y}=1 \mathrm{~m}$, and $v_{x}=v_{y}=0.01 \mathrm{~m}$ per sampling time step.

The same AIS configurations used by the CVM simulation are again used here. The AIS updating rate is every $60 \mathrm{~s}$ and the position measurement is subject to the noise modelled by a Gaussian white noise with zero mean and covariance diag $\{1,1\} \times 10^{2}$.

In Figure 6a, a trajectory tracking result is plotted. The ship makes its turn for 20 minutes and forms a final circle as seen by the plotted black line. AIS measurements are displayed by using small red circle markers and the positions provided by the KF are plotted by using blue star markers. It is evident that more accurate positions are provided by the KF at each AIS updating 


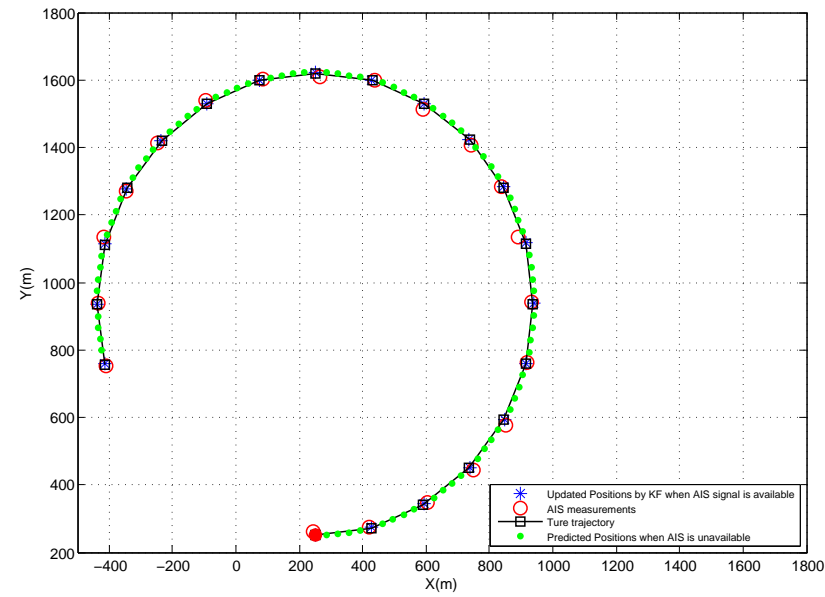

(a)

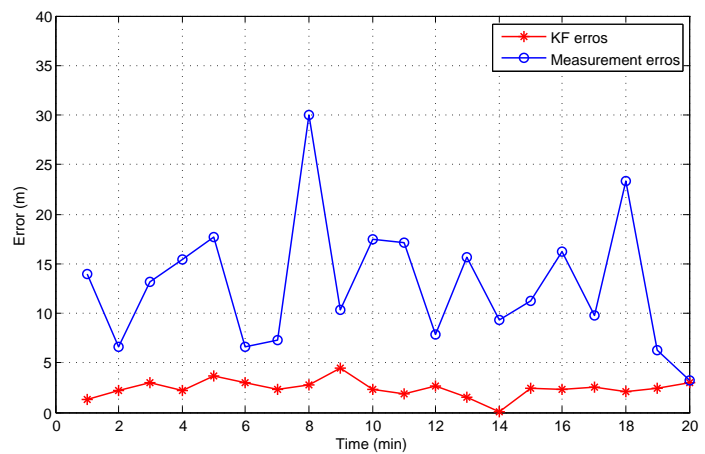

(b)

Figure 6. Simulation results of the trajectory tracking using the proposed KFTTA based on CTM. (a) Simulation results with true positions plotted with black square markers, observed positions plotted with red circle markers, positions provided by the KF plotted with start markers and predicted positions with green dot markers. (b) Comparison of position errors of measurement data and the data produced by the KFTTA.

point compared with the raw AIS measurement. In Figure 6b, the KF error (shown in red) is well below $5 \mathrm{~m}$; whereas, the measurement error is much larger with a maximum value approaching $30 \mathrm{~m}$. Such a dramatic error reduction is due to the fact that the CTM is better able to represent the ship's turning motion, and the KF relies more on the updated motion (Eq. 14) rather than the actual AIS measurement data to provide ship's positions.

Such an advantage can further assist the position prediction when AIS is not accessible. The turning motion of the ship can be reasonably well predicted with the ship's positions calculated using the Eq. 14. Predicted positions are shown as green dot markers in Figure 6a. It it evident that they are rather close to the true trajectory of the ship, which indicates accurate predictive navigation information of the moving ship has been provided even though AIS is not available.

\subsection{Trajectory tracking based on the CVM-CTM model}

In this test, the ship motion is represented by the CVM-CTM combined model and a simulation has been carried out to validate the trajectory tracking performance of the proposed IMM-CVMCTM filter. It is assumed that the ship starts its trajectory from the origin $(0,0)$ with the constant transverse speed of 4 knots. Also, in this simulation interest is in the tracking capability of the algorithm in a reliable maritime environment, the moving ship's information is thus updated every $4 \mathrm{~s}$ according to Table 1.

The CVM-CTM model consists of one CVM and two CTMs with the specific motion dynamics listed in Table 2. The ship first follows the CVM with a speed of 4 knots. From $21 \mathrm{~s}$, the ship 
Table 2. Ship motion dynamics based on CVM-CTM model.

\begin{tabular}{lllll}
\hline Trajectory section & Time steps & Motion model & Speed & Turning rate \\
\hline 1 & $0-20(\mathrm{~s})$ & CVM & 4 knots & 0 degrees $/ \mathrm{s}$ \\
2 & $21-100(\mathrm{~s})$ & CTM1 & 4 knots & 4 degrees $/ \mathrm{s}$ \\
3 & $101-120(\mathrm{~s})$ & CVM & 4 knots & 0 degrees $/ \mathrm{s}$ \\
4 & $121-180(\mathrm{~s})$ & CTM2 & 4 knots & 2 degrees $/ \mathrm{s}$ \\
5 & $181-200(\mathrm{~s})$ & CVM & 4 knots & 0 degrees $/ \mathrm{s}$ \\
\hline
\end{tabular}

starts to make the 1 st turn with a turning rate of $\omega_{1}=4$ degrees $/ \mathrm{s}$ for $80 \mathrm{~s}$. Such a model is denoted as the CTM1. At $101 \mathrm{~s}$, the ship switches back to the CVM motion and moves in a linear manner until $120 \mathrm{~s}$. At $121 \mathrm{~s}$, the second turn is made by the ship with the turning rate of $\omega_{2}=2$ degree/s and the model is denoted as CTM2. Finally, from 181 - $200 \mathrm{~s}$, the ship follows the CVM with the same speed of 4 knots. The real trajectory taken by the ship is plotted as the blue line shown in Figure 7 with 50 AIS measurements located along the trajectory plotted as the green dots. It is evident that the measurements suffer from noises resulting in deviations from the real trajectory. Such noise has been modelled by a Gaussian white noise sequence with zero mean and covariance diag $\{1,1\}$. The model transition probability between CVM, CTM1 and CTM2 is:

$$
p_{i j}=\left(\begin{array}{ccc}
0.8 & 0.1 & 0.1 \\
0.1 & 0.9 & 0 \\
0.1 & 0 & 0.9
\end{array}\right)
$$

which demonstrates that the models are able to transit from one to another except the crosstransition between CTM1 and CTM2.

Trajectory tracking results are plotted and compared in Figure 8. Tracking provided by the IMMCVM-CTM filter is shown in Figure 8d and tracking generated by the KF using CVM, CTM1 or CTM2 are presented in Figure 8a - 8c. It can be observed that tracking based on the CVM motion is able to provide more accurate results along straight line sections; whereas CTM based tracking performs better along curved sections. For example, the CTM2 based tracking (shown in Figure 8c) produces filtered trajectory close to the true trajectory along the first turn. However, more accurate tracking is provided by the IMM-CVM-CTM filter shown in Figure 8d. Table 3 lists the Mean Square Error (MSE) of the positions based upon the different models. It can be observed that CVM-CTM based tracking has the least MSE of $0.508 \mathrm{~m}$; whereas the highest MSE belongs to CTM2 based tracking. 


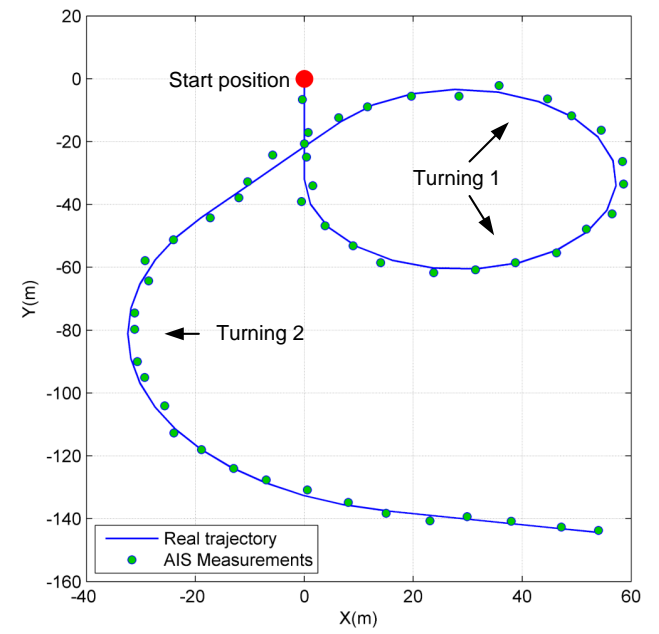

Figure 7. Real trajectory and the AIS measurements.

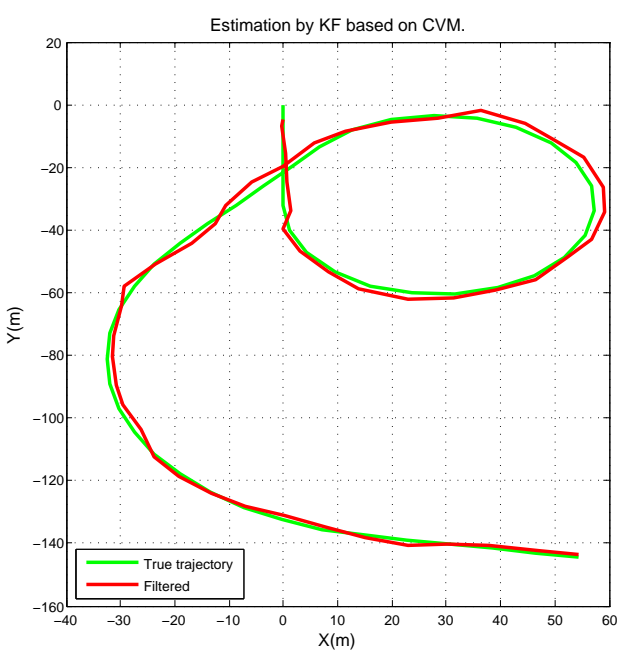

(a)

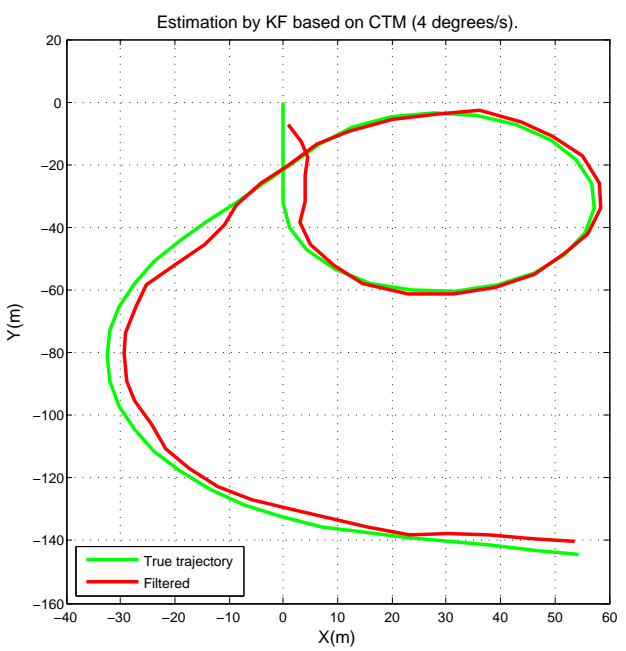

(c)

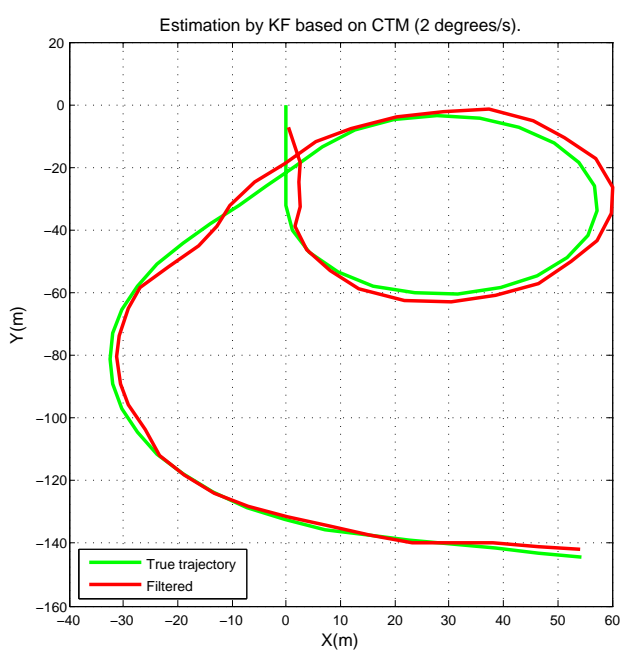

(b)

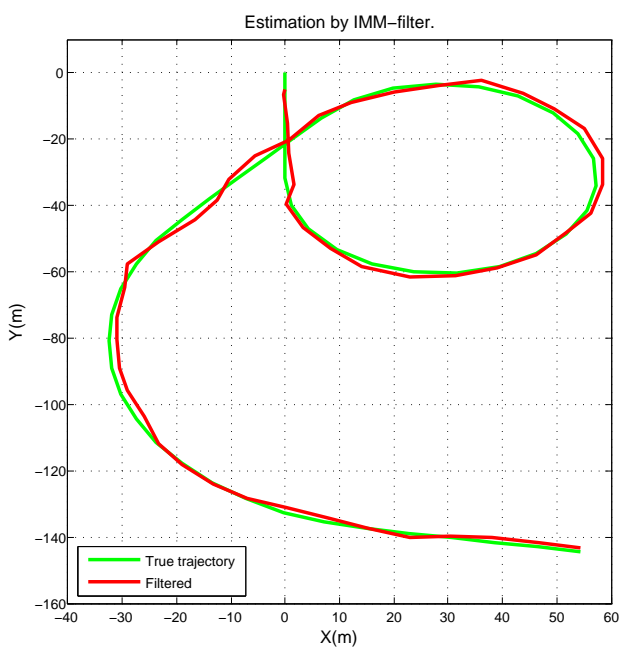

(d)

Figure 8. Trajectory tracking using different models. (a) Tracking based on the constant velocity model (CVM). (b) Tracking based on the coordinated turn model (CTM) with turning rate of 2 degrees per second. (c) Tracking based on the coordinated turn model (CTM) with turning rate of 4 degrees per second. (d) Tracking based on the IMM-filter. 
Table 3. Comparison of Mean Square Error (MSE) of positions based on different methods.

\begin{tabular}{ll}
\hline Model & MSE \\
\hline CVM & $0.659\left(\mathrm{~m}^{2}\right)$ \\
CTM1 & $1.39\left(\mathrm{~m}^{2}\right)$ \\
CTM2 & $1.89\left(\mathrm{~m}^{2}\right)$ \\
CVM-CTM & $0.508\left(\mathrm{~m}^{2}\right)$ \\
\hline
\end{tabular}

Figure 9 gives an illustration of how the model probabilities of the three different models change according to ship's movement. True probabilities are plotted as the green dashed lines indicate which model is being followed by the moving ship, i.e. value 1 represents the model has been used, and value 0 means it has not been used. The red line in each figure shows the filtered model probability representing the level of reliance of the current model. The higher the probability, the more likely the model will be used for trajectory tracking at the current time step. It can be observed that the red line has a similar trend to the green dashed line meaning the IMM-CVMCTM filter is able to make the right choice as to which model should be used. However, a small lag is reflected in Figure 9a-9c. This is because the IMM-CVM-CTM filter only has the position measurement of the ship and does not know the exact motion model taken by the ship. Therefore, the filter iteratively calculates the measurement residual (refer to $v_{k}^{j}$ in Eq. 19) of each model and gradually switches to the model with the least residual to complete the model transition. Such a transition process will take some time as the measurement residual does not change significantly or immediately.

\section{Conclusions}

In this paper, the problem of intelligent and robust moving ship tracking in uncertain environment has been addressed. The uncertainty in the maritime environment is primarily due to the measurement noises associated with shipborne devices such as the AIS. Also, the signal delay or signal lost problem in an area with severe environment conditions will cause problems for the vessel in detecting other moving ships. Therefore, to obtain more accurate information of moving obstacles, based on the basic motion of the ships a Kalman Filter based Trajectory Tracking Algorithm (KFTTA) has been proposed. The KFTTA is developed upon the constant velocity model (CVM) and coordinated turning model (CTM) of ships and a IMM-filter is implemented to integrate these two models to model more complex motion of a ship. From the simulation results, it has been shown that the KFTTA is able to largely reduce the measurement errors of AIS and to provide satisfactory predictions of moving ship's position when the AIS is unavailable. 


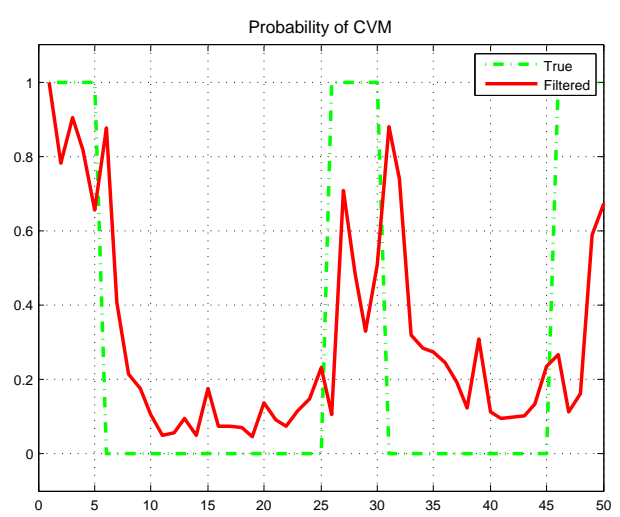

(a)

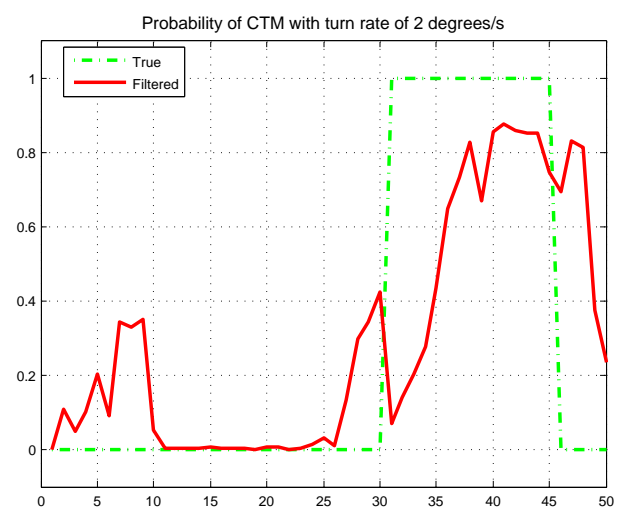

(b)

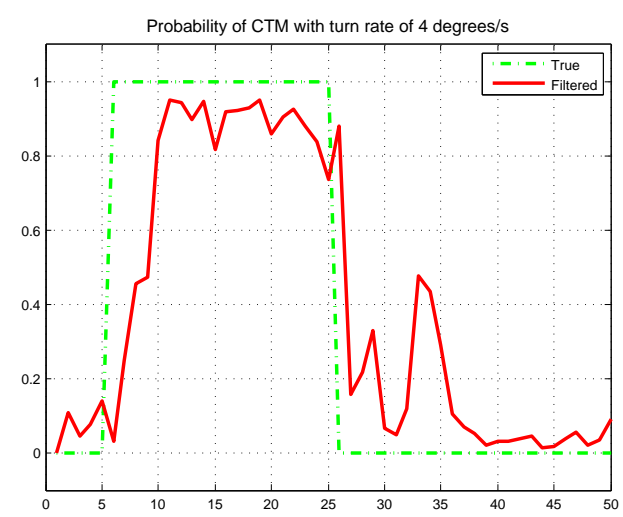

(c)

Figure 9. Model probabilities of different models. (a) Model probability of constant velocity model (CVM). (b) Model probability of coordinated turn model (CTM) with turning rate of 2 degrees per second. (c) Model probability of coordinated turn model (CTM) with turning rate of 4 degrees per second. 


\section{References}

Arroyo J. 2011. The automatic identification system: Then, now, and in the future. DTIC Document. Report No:

Bar-Shalom Y, Li XR, Kirubarajan T. 2004. Estimation with applications to tracking and navigation: theory algorithms and software. John Wiley \& Sons.

Campbell S, Abu-Tair M, Naeem W. 2014. An automatic colregs-compliant obstacle avoidance system for an unmanned surface vehicle. Proceedings of the Institution of Mechanical Engineers, Part M: Journal of Engineering for the Maritime Environment. 228(2):108-121.

Harati-Mokhtari A, Wall A, Brooks P, Wang J. 2007. Automatic identification system (AIS): data reliability and human error implications. Journal of navigation. 60(03):373-389.

Last P, Bahlke C, Hering-Bertram M, Linsen L. 2014. Comprehensive analysis of automatic identification system (AIS) data in regard to vessel movement prediction. Journal of Navigation. 67(05):791-809.

Li XR, Jilkov VP. 2000. Survey of maneuvering target tracking: dynamic models. In: AeroSense 2000. International Society for Optics and Photonics. p. 212-235.

Liu C, Chen X. 2013. Inference of single vessel behaviour with incomplete satellite-based ais data. Journal of Navigation. 66(06):813-823.

Liu Y, Liu W, Song R, Bucknall R. 2017. Predictive navigation of unmanned surface vehicles in a dynamic maritime environment when using the fast marching method. International Journal of Adaptive Control and Signal Processing. 31(4):464-488. 